\title{
Criminologie
}

\section{Regards croisés sur les processus de construction d'une identité professionnelle policière en France et au Québec}

\section{Marc Alain, Michel Rousseau et Dave Desrosiers}

Volume 46, numéro 2, automne 2013

Nouveaux regards sur les métiers de la sécurité

URI : https://id.erudit.org/iderudit/1020986ar

DOI : https://doi.org/10.7202/1020986ar

Aller au sommaire du numéro

\section{Éditeur(s)}

Les Presses de l’Université de Montréal

ISSN

0316-0041 (imprimé)

1492-1367 (numérique)

Découvrir la revue

Citer cet article

Alain, M., Rousseau, M. \& Desrosiers, D. (2013). Regards croisés sur les processus de construction d'une identité professionnelle policière en France et au Québec. Criminologie, 46(2), 43-67. https://doi.org/10.7202/1020986ar
Résumé de l'article

La question du réaménagement des représentations qu'un apprenant se fait du métier qu'il a choisi fait maintenant partie d'un corpus de connaissances connu sous l'appellation de la socialisation professionnelle. De manière générale, c'est a posteriori que ces réaménagements, leur amplitude et leur forme sont étudiés, en analysant les discours des représentants d'une ou de plusieurs professions. Il est, en effet, beaucoup plus rare de documenter ces mêmes réaménagements en temps réel, soit en suivant les parcours de socialisation au moment où ils se déroulent. Il est encore plus rare, également, qu'un dispositif de recherche longitudinal sur cohorte fasse l'objet d'une réplication dans un autre contexte. C'est cet exercice qui a été réalisé à dix ans d'intervalle en France, lieu de l'enquête originale, et au Québec par la suite. S'il ne nous appartient pas comme tel de tenter la comparaison des contextes français et québécois du métier de policier, en revanche, le fait de disposer ainsi de deux corpus de données d'enquête de socialisation professionnelle distincts nous donnera l'occasion de faire émerger les processus génériques communs de ce qui semble être plus spécifique à l'un ou l'autre des deux contextes. 


\title{
Regards croisés sur les processus de construction d'une identité professionnelle policière en France et au Québec
}

\author{
Marc Alain ${ }^{1}$ \\ Professeur titulaire \\ Département de psychoéducation, Université du Québec à Trois-Rivières \\ Directeur \\ Antenne du Centre international de criminologie comparée (CICC) à l'UQTR \\ marc.alain@uqtr.ca \\ Michel Rousseau \\ Professeur adjoint \\ Département de psychoéducation, Université du Québec à Trois-Rivières \\ michel.rousseau@uqtr.ca \\ Dave Desrosiers \\ Agent de milieu \\ Candidat à la maîtrise \\ Département de psychoéducation, Université du Québec à Trois-Rivières \\ dave.desrosiers@uqtr.ca
}

RÉSUMÉ - La question du réaménagement des représentations qu'un apprenant se fait du métier qu'il a choisi fait maintenant partie d'un corpus de connaissances connu sous l'appellation de la socialisation professionnelle. De manière générale, c'est a posteriori que ces réaménagements, leur amplitude et leur forme sont étudiés, en analysant les discours des représentants d'une ou de plusieurs professions. Il est, en effet, beaucoup plus rare de documenter ces mêmes réaménagements en temps réel, soit en suivant les parcours de socialisation au moment où ils se déroulent. Il est encore plus rare, également, qu'un dispositif de recherche longitudinal sur cohorte fasse l'objet d'une réplication dans un autre contexte. C'est cet exercice qui a été réalisé à dix ans d'intervalle en France, lieu de l'enquête originale, et au Québec par la suite. S'il ne nous appartient pas comme tel de tenter la comparaison des contextes français et québécois du métier de policier, en revanche, le fait de disposer ainsi de deux corpus de données

1. Marc Alain, Université du Québec à Trois-Rivières, Département de psychoéducation, 3351, boul. des Forges, C.P. 500, Trois-Rivières, (Québec) CANADA, G9A 5H7 
d'enquête de socialisation professionnelle distincts nous donnera l'occasion de faire émerger les processus génériques communs de ce qui semble être plus spécifique à l'un ou l'autre des deux contextes.

MOTS-CLÉS - socialisation professionnelle, culture professionnelle, culture policière, comparaison internationale, éthique policière.

\section{Introduction ${ }^{2}$}

Si les différents contextes historiques, sociaux, culturels et politiques peuvent circonscrire l'identité professionnelle attendue du policier de multiples façons, la question du devenir policier devient alors tout aussi multifactorielle puisqu'elle naît de l'influence entre une identité professionnelle générique et la multitude des identités individuelles de ceux et celles qui se destinent à devenir policiers; c'est le «qui je suis dans le qui nous sommes» de Legault (2003).

Or, quelle place peut être faite à ces différences individuelles dans une profession où l'adhésion au groupe est essentielle, voire, comme dans le cas des policiers, presque culturellement imposée? (Kappeler et al., 1998) La métaphore de l'entonnoir (Sherman, 1982; McNamara, 1999; Conti \& Nolan, 2005; Conti, 2006), rapportée dans Marcotte et Dion (2011), illustre très bien comment l'insertion professionnelle des policiers se fait en étapes successives, où le futur policier doit à chaque fois aménager son individualité au profit de comportements correspondant aux normes du groupe.

Ce sont ces processus, étudiés dans l'optique du travail, qui constituent le terrain des recherches menées sur le thème de la socialisation professionnelle (Dubar, 2000, 2010). Si cette question peut être abordée dans un cadre très large, puisqu'elle s'inscrit nécessairement sous l'angle de la professionnalisation et de la sociologie des professions, elle peut également être abordée de façon plus restreinte dans l'ici et maintenant vécu par des cohortes d'apprenants policiers. C'est l'angle privilégié dans cet article. Ainsi, alors que la professionnalisation peut se décliner en trois objets d'études distincts, soit celui de la professionnalisation des individus, des activités et des organisations (Roquet, 2012), c'est surtout sur l'interaction entre le processus de professionnalisation

2. Les auteurs remercient de leur collaboration les autorités de l'École nationale de police du Québec, sans qui la portion québécoise de cette recherche n'aurait pu être réalisée. Il va de soi que les opinions et commentaires exprimés ici n'engagent cependant que les auteurs du présent article. 
des individus et le fonctionnement des organisations que nous posons notre regard.

Ainsi, si l'apprenant policier est surtout confronté aux enjeux plus techniques et juridiques durant sa formation, nous pouvons supposer que la présence plus importante des enjeux politiques, économiques, administratifs ainsi que ceux des personnes visées par les interventions policières amènent nécessairement un réaménagement de sa pratique et de la représentation de sa profession. Ce sont sur ces réaménagements que nous allons maintenant porter notre attention, et ce, en tentant le jeu de la comparaison de données issues de deux enquêtes de cohortes menées tout d'abord en France (c'est l'enquête de cohorte Monjardet et Gorgeon), et dix ans plus tard, au Québec.

Après avoir brièvement présenté et comparé les modalités de formation policière en France et au Québec et expliqué les éléments de méthode de nos travaux, nous consacrerons la seconde partie de ce texte à une analyse descriptive de l'évolution des positions et des attitudes des recrues policières des deux échantillons au cours des différentes phases qui ont marqué les démarches de Monjardet et Gorgeon, d'une part, et les nôtres, d'autre part.

Plus précisément, les points de comparaison porteront d'abord sur différentes représentations des répondants relativement à leur métier et ce qui l'entoure, telles que les causes de la délinquance, la principale source de vigilance, l'aspect singulier du métier, la mission et les qualités du policier, ce qui est important dans le métier, vers qui se tourner en cas de problème et le désir de rester policier jusqu'à la retraite. Enfin, une comparaison des attitudes des répondants de deux cohortes sur la propension des policiers à utiliser la force et leur arme est réalisée, une comparaison qui permettra l'établissement de différentes classes.

\section{Les contextes de formation policière en France et au Québec}

L'accession à la formation des futurs policiers affectés à l'un ou l'autre des différents services rattachés à la Police nationale française débute par des concours nationaux de fonction publique. Les candidats admis au terme de ces concours peuvent dès lors s'inscrire à la formation initiale des gardiens de la paix, formation qui s'étend sur 12 mois et qui est dispensée dans onze écoles nationales de police pour les gardiens de la paix à travers l'Hexagone. C'est en fonction du classement 
atteint par le candidat ou la candidate, de même que des places disponibles, que les futures recrues amorceront le parcours de formation. La formation comprend une première période de cinq mois pendant laquelle les recrues abordent des éléments à la fois théoriques (maintien de l'ordre, droit pénal général et spécial, pour ne nommer que ces deux éléments) et pratiques (autodéfense, maniement d'armes, entraînement sportif, etc.). S'ensuit un stage de trois mois sur le terrain et, finalement, deux derniers mois de cours et de simulations. Cette formation initiale de gardiens de la paix ouvre la porte à l'ensemble des diverses affectations possibles à la police nationale, au sein de l'une ou l'autre des huit directions que compte la police nationale (l'inspection générale, les compagnies républicaines de sécurité, la police judiciaire, les renseignements intérieurs et la police aux frontières, pour ne nommer que celleslà). C'est cependant à la Direction générale de la sécurité publique qu'aboutit la très grande majorité des finissants des écoles de police. C'est lors de la dernière semaine de formation que les recrues recevront leur affectation où ils serviront pendant une période de deux à cinq ans à titre de gardiens de la paix stagiaires avant de recevoir la titularisation, équivalent québécois de la permanence d'emploi. Une comparaison sommaire avec le contexte québécois d'accession à la profession policière montre finalement que les deux cursus sont quantitativement semblables d'un endroit à l'autre. Il s'agit, en France, d'une formation de BAC général (formation équivalente à une formation générale collégiale au Québec) suivie d'une année en école de police, soit 13 ou 14 années de scolarité plus l'année en école, donc entre 14 et 15 ans de scolarité. Ce quantum correspond à celui des recrues policières du Québec, soit les 12 années cumulant le primaire et le secondaire, suivi des trois années en collège (ce sont les techniques policières ou encore les techniques auxiliaires de la justice, selon le collège) et des quinze semaines de stage à l'École nationale de police de Nicolet (ENPQ). On peut cependant se demander jusqu'à quel point ces cursus sont ou non qualitativement comparables. En effet, les recrues françaises bénéficient d'au moins un moment de stage au cours de leur formation, ce qui n'est pas le cas de leurs collègues du Québec. Les quinze dernières semaines de la formation qualifiante initiale à Nicolet sont présentées, il est vrai, généralement comme un «stage»; or, ce dernier est virtuel en ce sens que les recrues sont placées dans des situations préarrangées qui reposent sur les épaules de comédiens professionnels, dans des lieux spécifiquement construits à cette fin. En revanche, les recrues québé- 
coises ont bénéficié, pendant leurs trois années de collège, d'une formation technique très spécialisée, qui prépare très bien, en quelque sorte, le «moule» de ce qui est attendu de la fonction policière, et ce, dès 17 ou 18 ans. Les organisations policières québécoises n'offrent pas de structure d'accueil de stagiaires en raison, officiellement, du fait que les élèves de l'ENPQ (ou de l'un des collèges qui offrent les trois années de techniques policières) ne sont pas encore engagés et que, comme telles, les organisations policières ne peuvent garantir la santé et la sécurité au travail de personnes qui ne sont pas à leur emploi ${ }^{3}$. Mais cette absence de stage et de contacts réels avec l'intervention policière au Québec fait en sorte que les organisations qui vont embaucher les diplômés de l'ENPQ demandent beaucoup des quinze dernières semaines de formation des futures recrues. Ces organisations s'attendent en fait à ce que les recrues performent au plus haut niveau possible dès leur embauche et avec le niveau maximal de compétences attendues pour la totalité des tâches policières. En conséquence, les éléments abordés pendant les 15 semaines à l'ENPQ s'enchaînent les uns aux autres à un rythme rarement observé dans la réalité. Le programme développé par l'ENPQ tente de résoudre le dilemme créé par la nécessité de donner aux candidats toutes les compétences attendues en un court laps de temps, tout en maintenant un certain réalisme en ce qui a trait aux situations simulées qui sont offertes aux apprenants. On pourra comprendre que ce dilemme explique en partie le fait que plusieurs recrues se disent déçues par certains aspects plus répétitifs et routiniers de la fonction policière une fois qu'ils sont recrutés.

Mais il est un autre aspect de la formation policière au Québec qui pourra également contribuer au sentiment de déception exprimé par les recrues, il s'agit de la très forte compétition qui règne tout au long de ce parcours de formation, un phénomène qui, en vertu des modalités d'accès, pèse beaucoup moins sur les futures recrues françaises. La profession policière est au Québec, et ce, depuis maintenant une vingtaine d'années, très recherchée: on y fait de bons salaires, les taux de

3. Des contraintes d'ordre syndical constituent, plus officieusement, une autre raison de cet obstacle: en effet, les syndicats policiers veillent jalousement au principe que tout policier, peu importe son grade, sa fonction et son expérience, doit disposer des mêmes pouvoirs et prérogatives. On voit donc l'arrivée de stagiaires comme prétexte pour les hiérarchies à engager du personnel de seconde zone, moins rémunéré et qui pourrait être affecté à des tâches qui répugnent au personnel régulier, créant ainsi deux «classes» de policiers. 
placement atteignent près de cent pour cent et on peut prétendre à la retraite après 25 ans de service, soit à un âge où une seconde carrière est tout à fait envisageable, ce qui ouvre la possibilité de cumuler un nouveau salaire et une rente de retraite déjà fort généreuse. Il est dès lors peu surprenant de constater qu'à l'entrée du cursus, quatre demandes sur cinq sont rejetées et que seulement un candidat sur vingt parviendra à décrocher le diplôme ${ }^{4}$. Si l'on peut penser que ce niveau élevé de compétition garantira que seuls les meilleurs candidats et candidates passeront au travers du processus, on pourra également penser qu'en revanche, il se créera un niveau d'attente tout aussi élevé, susceptible d'engendrer son lot de déceptions, voire de frustrations. Ce sont là, théoriquement du moins, des déceptions beaucoup moins susceptibles de frustrer les recrues en France, essentiellement en raison du processus des entrées latérales qui mène aux fonctions hiérarchiques supérieures.

\section{Aspects méthodologiques des deux enquêtes}

Contextes de passation des questionnaires des deux enquêtes de socialisation professionnelle, au Québec et en France

Bien que la plupart des efforts consacrés à l'étude des processus de socialisation professionnelle des policiers reposent sur les propos tenus, a posteriori, par des policiers de carrière décrivant leur période d'entrée en fonction (Manning \& Van Maanen, 1978; Crank, 1998), quelques chercheurs avaient plutôt abordé la question en suivant des recrues pendant les premiers moments de leur intégration professionnelle, de McNamara (1967) à Chan (2003), en passant par Van Maanen (1973, 1974, 1977) et par Fielding (1988). Certains des chocs de la réalité que ces chercheurs décrivent se retrouvent à des degrés divers dans les réponses données par les participants français et québécois sur lesquelles nous allons nous pencher ici.

4. Un des effets notables de ce contingentement est d'avoir considérablement gonflé de policières les rangs de cette profession traditionnellement masculine. Comme on sait qu'en général les jeunes femmes sont nettement plus assidues aux études que leurs confrères masculins, elles affichent des rendements scolaires qui leur ouvrent plus facilement la porte des programmes contingentés. Ce qui fait donc que sans même s'être dotées de politiques officielles de discrimination positive, les organisations policières québécoises ont vu leurs rangs être occupés par près de 40 pour cent de policières au cours des quelques dernières années. 
La démarche réalisée au Québec s'inspire très largement des travaux menés en France par Monjardet et Gorgeon (1992, 1993, 1996, 1999, $2004)^{5}$. Leur principal objectif, tout comme le nôtre, consistait à étudier le processus d'intégration professionnelle au fur et à mesure qu'il se déroule, et ce, avec un échantillon suffisamment grand pour y distinguer les différentes modalités d'adaptation et leurs liens avec les attitudes et prédispositions exprimées par les participants en tout début d'étude. Si Monjardet et Gorgeon ont pu compter sur la participation initiale de 1167 répondants, l'échantillon québécois, quoique plus modeste, demeure tout à fait représentatif de la population des recrues policières de la période des réponses au premier temps de l'enquête longitudinale. En effet, nous avons été en mesure de recueillir les réponses de 734 des 744 étudiants à l'ENPQ pendant l'année scolaire 2001-2002 ${ }^{6}$. Bien que les deux groupes soient initialement à peu près comparables en termes de nombre, les moments de passation du questionnaire, eux, ne le sont que partiellement. En effet, les trois premiers temps de l'enquête française se sont déroulés pendant les 12 mois de la formation en école de police, soit lors de la toute première semaine de formation pour le temps 1 , après la période de formation théorique de cinq mois et au retour du congé des fêtes pour le temps 2 , et pendant leur dernière semaine en école, juste avant la notification de leur affectation pour le temps 3. Les participants québécois, quant à eux, ont tous été abordés au milieu de leurs 15 semaines de formation, soit à la huitième semaine pour le temps

5. Dominique Monjardet était parfaitement au courant de l'existence de l'enquête québécoise dont il avait lu, commenté et apprécié la version du questionnaire utilisé; cette version demeurait par ailleurs aussi fidèle que possible au questionnaire original et seuls des termes uniques au contexte français - les grades, notamment, de même que des expressions juridiques très spécifiques - avaient été adaptés au contexte québécois que Monjardet connaissait très bien. Le premier auteur du présent texte eut également l'occasion de s'entretenir longuement et à deux reprises - 2003 et 2005 - avec le chercheur français quant à la possibilité d'une publication conjointe des résultats comparés des deux enquêtes, un projet malheureusement emporté par le décès de Dominique Monjardet en 2006. Nous avons cependant été en mesure de tenter une forme d'hommage à l'héritage de Monjardet en codirigeant, avec G. Pruvost (CESDIP), un numéro spécial de la revue Déviance et Société (2011, vol. 35 [3]) portant sur la thématique de la socialisation professionnelle des policiers.

6. Notons à cet égard, et contrairement au cas de la France où l'on retrouve onze écoles de police, que l'ENPQ constitue la seule voie d'entrée au Québec pour devenir policier (même si on compte les recrues ayant fait leur formation initiale qualifiante à l'école de la Gendarmerie royale du Canada, à Regina, qui ne constituent, et pas toutes les années, que quelques recrues tout au plus). Ceci fait en sorte que le bassin de répondants de départ représente la quasi-population de ceux et celles qui vont devenir policiers à partir de la fin de leur formation de quinze semaines à Nicolet cette année-là. 
1. C'est précisément un an après leur sortie d'école de police que les recrues québécoises ont été abordées pour répondre au questionnaire du temps 2. Le temps 3 de l'enquête, quant à lui, s'est déroulé un an après le second. À l'analyse des réponses données au temps 3, il est ressorti que certains schémas de réponse semblaient commencer à s'établir pour plusieurs répondants, et ce, sans compter certains commentaires écrits de ces mêmes répondants, à savoir que l'on commençait à trouver la démarche bien pénible. La décision fut alors prise de décaler la quatrième passation de deux années complètes. Le temps 4 de l'enquête se situait donc entre la quatrième et cinquième année de service des recrues québécoises ${ }^{7}$. Pour ce qui est de l'enquête québécoise, dans la suite de ce que nous avions constaté à la phase précédente à propos des réticences croissantes à répondre au questionnaire ${ }^{8}$ et, surtout, de l'investissement considérable nécessaire pour s'assurer d'un groupe de répondants suffisamment nombreux, la décision fut prise de mettre fin à l'étude 9 . Au constat de la décision de mettre fin à l'enquête québécoise après seulement quatre passations, on pourra avancer, sans aucunement discréditer les travaux accomplis en France, qu'il est nettement plus difficile au Québec de retrouver les participants policiers à des études longitudinales, étant donné que ces participants ont été

7. Cet étirement correspond au fait que les 734 répondants de la première vague ont répondu au questionnaire en trois temps différents durant l'année 2001-2002. En effet, la capacité d'accueil de l'école de Nicolet étant limitée, les élèves sont répartis en trois groupes qui vont se succéder les uns après les autres pour leur stage de 15 semaines. Ainsi, au moment où le troisième groupe répondait au questionnaire du temps 1 , il était pratiquement temps de faire passer le questionnaire du temps 2 aux répondants du premier de ces trois groupes.

8. En témoignent ces trois exemples tirés des commentaires écrits par des répondants: "Je trouve ce sondage de plus en plus lourd à compléter. Est-ce que ça achève tout çà? En tout cas, j'espère que des améliorations concrètes sont effectuées dans notre formation continue grâce à ce sondage. Merci.» Extrait $n^{\circ} 4$; «Questionnaire trop long.» Extrait $\mathrm{n}^{\circ} 12$; «Trop Long. Si vous voulez plus de candidats, payez...» Extrait $\mathrm{n}^{\circ} 16$.

9. Le questionnaire de la phase 4 a été envoyé une première fois et a généré un taux de réponse beaucoup trop faible d'à peine $30 \%$ (ces taux étaient de $55 \%$ en phase 2 et de $43 \%$ en phase 3). Une campagne de sensibilisation a alors été lancée dans tous les corps de police du Québec afin de rappeler à ceux et celles qui avaient été les participants de départ (les cohortes 19 à 30 de l'ENPQ) l'importance de participer à l'exercice. Finalement, décision fut prise en fin de 2007 - début 2008 de contacter personnellement ceux et celles des répondants qui avaient été jusqu'ici les plus fidèles à répondre et qui ne l'avaient pas encore fait pour la quatrième passation. Notons que le personnel affecté à cette tâche fastidieuse s'est fait dire à cette occasion qu'on répondrait au questionnaire à condition que ce soit la dernière fois. 
engagés par pas moins de 30 organisations policières différentes ${ }^{10}$. Ceci dit, il n'en demeure pas moins tout à fait remarquable que Monjardet et Gorgeon aient réussi à conserver un tel niveau de fidélité de la part des répondants et que 10 années après la première vague, ce soit $46 \%$ des participants de départ qui collaboraient encore. Le tableau 1 permet de comparer les divers moments de passation en fonction des deux enquêtes, de même que les variations des proportions de répondants d'une vague à l'autre.

\section{TA B LE A U 1}

Les moments de passation des questionnaires, le laps de temps entre chaque passation et le nombre de participants (pourcentage par rapport au nombre initial de participants en première passation), au Québec et en France

\begin{tabular}{|c|c|c|c|c|c|c|}
\hline & $\mathrm{T} 1$ & $\mathrm{~T} 2$ & T3 & T4 & T5 & T6 \\
\hline $\begin{array}{l}\text { Québec } \\
\text { (de 2001- } \\
2002 \text { à } \\
2007- \\
2008 \text { ) }\end{array}$ & $\begin{array}{l}7 \\
\text { semaines } \\
\text { avant } \\
\text { l'entrée } \\
\text { en } \\
\text { fonction } \\
\mathrm{n}=734\end{array}$ & $\begin{array}{l}12 \text { mois } \\
\text { après } \\
\text { l'entrée } \\
\text { en } \\
\text { fonction } \\
\mathrm{n}=398 \\
(54 \%)\end{array}$ & $\begin{array}{l}24-36 \\
\text { mois } \\
\text { après } \\
\text { l'entrée } \\
\text { en } \\
\text { fonction } \\
\mathrm{n}=316 \\
(43 \%)\end{array}$ & $\begin{array}{l}48-60 \\
\text { mois } \\
\text { après } \\
\text { l'entrée } \\
\text { en } \\
\text { fonction } \\
\mathrm{n}=281 \\
(38 \%)\end{array}$ & N.A. & N.A. \\
\hline $\begin{array}{l}\text { France } \\
\text { (de 1992 } \\
\text { à 2002) }\end{array}$ & $\begin{array}{l}\text { Arrivée } \\
\text { en école } \\
\text { de police } \\
\mathrm{n}=1166\end{array}$ & $\begin{array}{l}\text { Au } \\
6^{\mathrm{e}} \text { mois } \\
\text { de } \\
\text { formation } \\
\mathrm{n}=1157 \\
(99 \%)\end{array}$ & $\begin{array}{l}\mathrm{Au} \\
12^{\mathrm{e}} \text { mois } \\
\text { de } \\
\text { formation } \\
\mathrm{n}=1109 \\
(95 \%)\end{array}$ & $\begin{array}{l}12 \text { mois } \\
\text { après } \\
\text { l'entrée } \\
\text { en } \\
\text { fonction } \\
\mathrm{n}=684 \\
(59 \%)\end{array}$ & $\begin{array}{l}6 \text { ans } \\
\text { après } \\
\text { l'entrée } \\
\text { en } \\
\text { fonction } \\
n=610 \\
(52 \%)\end{array}$ & $\begin{array}{l}10 \text { ans } \\
\text { après } \\
\text { l'entrée } \\
\text { en } \\
\text { fonction } \\
\mathrm{n}=530 \\
(46 \%)\end{array}$ \\
\hline
\end{tabular}

Les éléments illustrés au tableau 1 nous montrent quelles sont les possibilités d'appariement des données des deux enquêtes. Étant donné que la première vague de questionnement des recrues québécoises se déroule quelques semaines avant l'entrée en fonction (c.-à-d. l'embauche par un corps de police), elle correspond en fait beaucoup plus au troisième temps de l'enquête française qu'au premier. Le second temps de l'enquête québécoise (T2), quant à lui, correspond à la quatrième passation des répondants français et, finalement, le dernier temps de l'enquête québécoise (T4) correspond approximativement au cinquième moment de l'enquête réalisée auprès des recrues de l'école de police de Paris.

10. Et c'est également sans compter le fait qu'au cours des premières années de carrière, les recrues peuvent changer d'employeur à plus d'une reprise. 


\section{La question de la représentativité}

Deux précautions s'imposent ici. Il va de soi, en premier lieu, que ces 281 répondants ne peuvent en aucun cas être considérés comme représentatifs de la population totale des policiers québécois. Ils pourraient cependant l'être de ceux et celles qui ont maintenant entre 5 et 6 ans d'expérience. Nous devons toutefois convenir que ce n'est là qu'un postulat théorique. Mais, compte tenu de la quasi-exclusivité de l'ENPQ en matière de formation initiale en patrouille gendarmerie au Québec (l'exception étant des policiers, cas plutôt rares, qui ont été formés à la GRC), nos 734 recrues de départ devraient normalement représenter une majorité des policiers québécois qui ont été engagés au sein des corps policiers entre avril 2002 et janvier 2003.

Mais, pour autant qu'en termes strictement statistiques ces mêmes résultats soient significatifs, la question se posera toujours quant à leur validité. C'est qu'il nous faut démontrer, en fait, jusqu'à quel point les réponses qui nous sont fournies par les participants reflètent bel et bien les attitudes et positions de l'ensemble des sujets de départ (les répondants de la toute première administration du questionnaire) et ne sont pas, en d'autres termes, uniquement le reflet des attitudes de ceux et celles qui acceptent de répondre au questionnaire. C'est en utilisant deux moyens différents expliqués dans deux contributions précédentes consacrées à l'analyse des réponses de la seconde phase de l'étude (Alain \& Baril, 2005a, 2005b) que nous sommes en mesure de prétendre à la validité globale des résultats. Dans le cas de l'enquête réalisée en France, de telles précautions se seraient avérées bien inutiles.

Il va de soi qu'un travail de mise en comparaison, a posteriori, de données qui n'avaient pas nécessairement été pensées à cette fin et, par-dessus le marché, à plus de dix ans d'intervalle dans des contextes culturels et organisationnels extrêmement différents, impliquera de grandes précautions quant à ce qu'il permet d'affirmer. Si tant est, donc, que les deux contextes d'étude sont fort différents, il n'en demeure pas moins que nous avons l'occasion de suivre ici, et en parallèle, les parcours de socialisation professionnelle de deux échantillons de policiers. La principale question sera alors de voir jusqu'à quel point ces parcours se ressemblent ou non, et ce, en mesurant les attitudes et opinions des répondants à peu près aux mêmes étapes. 


\section{Les questionnaires}

Les questionnaires utilisés dans l'étude québécoise sont similaires à ceux de l'étude française. Ils contiennent plus d'une centaine de questions portant sur une diversité de sujets reliés à la réalité policière. Comme dans le dispositif original de l'enquête française, la majorité des questions étaient répétées d'un temps à l'autre de l'administration, permettant ainsi des comparaisons des trajectoires évolutives des répondants. L'outil développé pour l'enquête québécoise inclut cependant une échelle mesurant le niveau d'éthique des répondants. Cette échelle est composée de 19 items représentant des situations problématiques sur le plan éthique. L'item "exagérer une accusation afin de mettre quelqu'un hors d'état de nuire est acceptable» en constitue un bon exemple. Chaque item présente une échelle de réponses de type Likert en 5 points $(1=$ Tout à fait en désaccord à $5=$ Tout à fait d'accord $)$. Tous les items ont été recodés de telle sorte que plus le score est élevé, plus le niveau d'éthique théorique du répondant l'est aussi. Les scores à cette échelle peuvent varier entre 19 et 95 points. Cette mesure a été vérifiée aux quatre temps de mesure de l'enquête québécoise. La valeur du coefficient alpha de Cronbach est de 0,94 au temps 1, 0,87 au temps 2 et 0,89 aux temps 3 et 4 , ce qui situe l'échelle à des niveaux de cohérence interne tout à fait acceptables (Hogan et al., 2012).

\section{Modèles et analyses statistiques privilégiés}

Des analyses descriptives ont tout d'abord été réalisées afin de présenter les caractéristiques des deux échantillons, ainsi que leurs réponses à certaines questions qui détaillent les représentations du métier. Les questions concernant les justifications à l'usage de l'arme et à l'usage de la force ont constitué la thématique principale touchant les représentations que se font les répondants du métier policier. De plus, comme ces questions étaient identiques pour les questionnaires des deux enquêtes, elles constituaient d'autant une bonne base de comparaison et des deux échantillons et des évolutions respectives des opinions dans un cas comme dans l'autre. Cependant, compte tenu des disparités entre ces deux enquêtes, aucune analyse statistique n'a pu être mise à profit pour faire ressortir mathématiquement les points de convergence et de divergence entre les évolutions respectives des deux groupes. Pour identifier les sous-populations de répondants qui optent pour les mêmes 
réponses sur les questions portant sur l'utilisation de la force et de leur arme, nous avons eu recours à une analyse de classes latentes (McCutcheon, 1987; Muthen \& Muthen, 2000). Ce type d'analyse avait également été retenu dans l'étude de Coulangeon (2012), il permet de regrouper les répondants en fonction de leurs schémas de réponses aux questions afin d'identifier ceux présentant des schémas de réponses similaires, donc qui partagent un point de vue commun. Lanalyse des classes latentes présente l'avantage de fournir des résultats statistiques à partir desquels il est possible de choisir le meilleur modèle représentant les données, et ce, contrairement à d'autres approches qui laissent un choix trop arbitraire aux chercheurs. Le choix du meilleur modèle est basé sur la valeur de l'indice de BIC («Bayesian Information Criterion»). Le modèle retenu est celui pour lequel cet indice est le plus faible. Par la suite, le modèle répartit les répondants selon les classes latentes identifiées et calcule une probabilité que ces répondants donnent une réponse affirmative à chacune des questions portant sur l'utilisation de la force ou de l'arme. Les tableaux présentés dans cet article rapportent ces probabilités. Le nom donné à chacune des classes dépend alors des probabilités de réponses aux questions. Enfin, pour l'échantillon de répondants québécois, une dernière analyse met en relation les classes latentes retenues et leur niveau d'éthique, une analyse malheureusement impossible à calquer sur les données de l'enquête française qui n'abordait pas cette question de manière aussi détaillée. Des tests T ont été utilisés pour comparer la moyenne du score éthique des répondants de la première classe à celle des répondants de la deuxième classe. Cette même analyse a ensuite été répétée pour chacun des quatre temps de mesure.

\section{Résultats}

\section{Description de l'échantillon}

L'échantillon de policiers québécois est composé de $58 \%$ d'hommes. Ils sont en majorité âgés de 21 à 25 ans (tableau 2). Une très grande proportion $(92 \%)$ provient de la filière des programmes de formation avec un diplôme d'études collégiales (DEC) et seulement 7 \% n'auraient pas refait d'études en technique policière. Pour sa part, l'échantillon de policiers français compte une plus grande proportion d'hommes, avec $81 \%$ (tableau 3). Ils sont aussi en majorité âgés de 21 à 25 ans. Un peu plus de $40 \%$ des répondants ont été policiers ou gendarmes auxiliaires 
TABLEAU 2

Description de l'échantillon des répondants québécois

\begin{tabular}{|l|r|r|}
\hline & $\mathrm{n}$ & $\%$ \\
\hline Sexe & & \\
Homme & 417 & 58,2 \\
Femme & 300 & 41,8 \\
\hline Groupe d'âge & & \\
Moins de 21 ans & 92 & 12,8 \\
De 21 à 25 ans & 500 & 69,4 \\
De 26 à 30 ans & 91 & 13,5 \\
Plus de 30 ans & 31 & 4,3 \\
\hline Formation & & \\
DEC & 664 & 92,1 \\
AEC & 57 & 7,9 \\
\hline Refaire des études en T.P. & & \\
Oui & 668 & 92,8 \\
Non & 52 & 7,2 \\
\hline
\end{tabular}

TABLEAU 3

Description de l'échantillon des répondants français

\begin{tabular}{|l|r|r|}
\hline & $\mathrm{n}$ & $\%$ \\
\hline Sexe & & \\
Homme & 899 & 81,1 \\
Femme & 209 & 18,9 \\
\hline Groupe d'âge & & \\
Moins de 21 ans & 124 & 11,2 \\
De 21 à 25 ans & 806 & 73,1 \\
De 26 à 30 ans & 173 & 15,7 \\
\hline Service national P.A ou G.A. & & \\
Policier auxiliaire & 362 & 32,6 \\
Gendarme auxiliaire & 87 & 7,8 \\
Non concerné & 660 & 59,5 \\
\hline Se représenter au concours & & \\
Oui & 979 & 88,8 \\
Non & 124 & 11,2 \\
\hline
\end{tabular}


TA B LEA U 4

Évolution des opinions comparées à des temps équivalents entre l'échantillon des répondants québécois et celui des répondants français (caractères en italique)

\begin{tabular}{|c|c|c|c|c|c|c|}
\hline & \multicolumn{2}{|c|}{$\begin{array}{c}1^{\text {er }} \text { temps } \\
\text { de comparaison }\end{array}$} & \multicolumn{2}{|c|}{$\begin{array}{c}2^{\mathrm{e}} \text { temps } \\
\text { de comparaison }\end{array}$} & \multicolumn{2}{|c|}{$\begin{array}{c}3^{\mathrm{e}} \text { temps } \\
\text { de comparaison }\end{array}$} \\
\hline & $\mathrm{T} 1(0)$ & $T 3(F)$ & $\mathrm{T} 2(0)$ & $T 4(F)$ & $\mathrm{T} 4(0)$ & $T 5(F)$ \\
\hline \multicolumn{7}{|l|}{ Cause de la délinquance } \\
\hline Économique et sociale & 54,0 & 57,3 & 46,7 & 39,2 & 24,1 & 25,7 \\
\hline Crise des institutions & 6,2 & 4,1 & 2,9 & 4,0 & 1,4 & 4,4 \\
\hline Immigrés & 0,7 & 15,2 & 1,8 & 22,0 & 4,7 & 13,3 \\
\hline Tolérance de la justice & 13,8 & 11,0 & 23,0 & 21,8 & 41,7 & 25,2 \\
\hline Démission des parents & 25,3 & 12,4 & 25,6 & 13,0 & 28,1 & 31,4 \\
\hline \multicolumn{7}{|l|}{ Vigilance auprès } \\
\hline Automobilistes & 12,9 & 1,9 & 14,3 & 2,8 & 6,9 & 2,3 \\
\hline Drogués & 11,1 & 12,5 & 19,5 & 14,3 & 21,4 & 11,1 \\
\hline Jeunes & 29,5 & 35,5 & 31,3 & 27,6 & 31,2 & 45,8 \\
\hline Immigrés & 2,5 & 13,8 & 3,6 & 21,5 & 4,7 & 14,6 \\
\hline Trafiquants & 28,5 & 31,9 & 17,7 & 27,5 & 26,8 & 17,9 \\
\hline Autres truands & 15,4 & 4,4 & 13,5 & 6,3 & 9,1 & 8,1 \\
\hline $\begin{array}{l}\text { Policier est un métier comme } \\
\text { un autre }\end{array}$ & 15,4 & 34,7 & 16,6 & 36,3 & 12,1 & 22,8 \\
\hline \multicolumn{7}{|l|}{ Mission du policier } \\
\hline Faire respecter la loi & 31,6 & 48,2 & 38,0 & 45,9 & 44,2 & 47,9 \\
\hline Secourir & 65,6 & 21,6 & 56,3 & 28,0 & 44,6 & 19,7 \\
\hline Protéger les institutions & 0,0 & 5,5 & 0,3 & 6,0 & 0,4 & 6,2 \\
\hline Lutter contre les délinquants & 2,8 & n.a. & 5,4 & n.a. & 10,9 & n.a. \\
\hline Prévenir la délinquance & n.a. & 18,9 & n.a. & 20,1 & n.a. & 26,1 \\
\hline \multicolumn{7}{|l|}{ Qualité du policier } \\
\hline Honnête & 62,6 & 61,3 & 47,3 & 58,8 & 45,7 & 51,2 \\
\hline Courageux & 0,4 & 0,8 & 1,0 & 2,1 & 1,1 & 2,3 \\
\hline Débrouillard & 11,4 & 2,6 & 23,3 & 3,8 & 20,3 & 3,9 \\
\hline Ouvert & 11,8 & 14,7 & 8,5 & 10,7 & 7,2 & 8,0 \\
\hline Discipliné & 6,4 & 2,3 & 4,9 & 3,2 & 3,3 & 3,6 \\
\hline Nerfs solides & 1,4 & 4,1 & 0,5 & 7,3 & 3,6 & 18,6 \\
\hline Observateur & 6,0 & 12,4 & 14,5 & 14,1 & 18,8 & 12,3 \\
\hline \multicolumn{7}{|l|}{ Le plus important dans le métier } \\
\hline Travail intéressant & 30,1 & 45,3 & 19,6 & 36,6 & 23,4 & 35,1 \\
\hline Bonne ambiance & 28,8 & 14,5 & 40,1 & 22,7 & 39,6 & 29,7 \\
\hline Compter sur ses collègues & 34,0 & 35,0 & 36,4 & 37,0 & 36,0 & 31,7 \\
\hline Respecter les règlements & 7,1 & 5,1 & 3,9 & 3,7 & 1,1 & 3,4 \\
\hline \multicolumn{7}{|c|}{ En cas de problème sur qui compter } \\
\hline Collègues & 54,1 & 31,3 & 58,0 & 44,3 & 56,0 & 32,4 \\
\hline Famille & 27,1 & 33,4 & 18,5 & 24,2 & 20,9 & 40,0 \\
\hline Syndicats & 1,8 & 17,6 & 9,9 & 14,4 & 11,2 & 8,1 \\
\hline Hiérarchie & 4,4 & 8,5 & 3,1 & 10,2 & 2,2 & 6,1 \\
\hline Amis & 12,6 & 9,2 & 10,4 & 6,9 & 9,7 & 13,5 \\
\hline $\begin{array}{l}\text { Je serai policier jusqu'à } \\
\text { la retraite }\end{array}$ & 88,7 & 69,3 & 86,0 & 76,0 & 79,8 & 67,2 \\
\hline
\end{tabular}


durant leur service national. Enfin, seulement $11 \%$ ont affirmé qu'ils ne se représenteraient pas au concours d'entrée.

\section{Représentations du métier et déceptions}

Il semble exister certains points de convergence et de divergence au niveau des représentations du métier chez les policiers québécois et les policiers français. Le tableau 4 indique une évolution identique pour les deux groupes en ce qui a trait à leur opinion par rapport à la principale cause de la délinquance est observée. Au premier temps de mesure, $54 \%$ des répondants québécois et $57 \%$ des répondants français ont identifié «la situation économique et sociale» comme principale cause de la délinquance alors qu'au dernier temps de mesure, cette proportion est de $24 \%$ pour les Québécois et de $26 \%$ pour les Français. Lors du dernier temps de mesure, la «tolérance de la justice» a été identifiée comme principale cause de la délinquance par $42 \%$ des Québécois et par $25 \%$ des Français. Un point de divergence apparaît entre les deux groupes en ce qui concerne les «immigrés» qui sont perçus comme principale cause de la délinquance par une proportion plus élevée de policiers français que québécois. De même, une proportion plus élevée de Français affirment devoir être plus vigilants auprès des «immigrés», et ce, comparativement aux Québécois.

Le métier de policier est vu comme étant un métier comme les autres pour une plus grande proportion de répondants français que québécois. En effet, la proportion de policiers français qui sont en accord avec l'énoncé «policier est un métier comme un autre» varie entre $35 \%$ au premier temps de comparaison et $23 \%$ au troisième temps alors que pour les policiers québécois, ces proportions passent de $15 \%$ à $12 \%$. La vision de la mission du policier est aussi différente dans les deux groupes. Chez les répondants québécois, $66 \%$ choisissent la réponse «secourir» à la question de savoir quelle est «la mission première du policier» au premier temps de comparaison, cette réponse n'étant plus choisie que par $45 \%$ des répondants au dernier temps de mesure. À l'inverse, la proportion de répondants qui affirment que «la mission première du policier» est de «faire respecter la loi» ou de «lutter contre les délinquants» augmente régulièrement et significativement au fils des ans. Une proportion moins élevée de répondants français a opté pour la réponse «secourir», elle varie entre $22 \%$ au premier temps et $20 \%$ lors du dernier temps de comparaison. 
Une certaine convergence est observée en ce qui a trait à l'opinion relative à «la qualité première du policier». Dans les deux groupes, l'honnêteté est perçue comme étant la qualité la plus importante. Au premier temps de mesure, près des deux tiers des répondants québécois et français ont opté pour cette réponse. Il est aussi observé que pour les deux groupes, cette proportion diminue graduellement chaque année. Pour les policiers québécois, la proportion de répondants qui optent pour les réponses «d'être débrouillard» ou «d'être observateur» a par contre augmenté tandis que chez les policiers français, c'est la réponse «avoir les nerfs solides» qui a le plus augmenté.

On observe également que le tiers des répondants québécois ont affirmé à chaque temps de mesure que le plus important dans le métier était de compter sur les collègues. Une proportion du même ordre est observée chez les Français en plus de présenter une stabilité d'un temps à l'autre de l'enquête. De plus, la proportion de policiers qui répondent qu'en cas de problème, ils compteraient sur leurs collègues est relativement stable d'un temps de passation à l'autre et ce, autant chez les Québécois que les Français, bien que cette proportion varie entre $54 \%$ et $56 \%$ pour les répondants québécois et entre $31 \%$ et $32 \%$ pour les français.

Enfin, une plus grande proportion de policiers québécois que français affirment qu' ils vont être policiers jusqu'à la retraite, mais dans les deux cas, une légère diminution est observée entre le premier et le troisième temps de comparaison.

\section{Usage de la force et usage de l'arme}

En regard des motifs au nom desquels l'usage de la force et l'usage de l'arme de service se justifient, le modèle de classification qui répond le mieux aux critères méthodologiques (en fonction de la qualité de l'ajustement et de la parcimonie) permet de regrouper les répondants québécois en deux classes distinctes. Rappelons ici que pour chacun des choix de justification du recours à la force et à l'arme, les participants répondent par oui ou par non; dans les tableaux 5 et 7 , plus le coefficient est élevé, plus grande est la possibilité que les répondants choisissent «oui» comme réponse. Les répondants de la classe 1 ont donc tendance à avoir recours à la force ou à leur arme dans une variété de situations tandis que ceux de la classe 2 réservent l'usage de la force ou de leur arme pour des situations très précises, soit «vis-à-vis d'un 
TABLEA U 5

Description des classes pour les policiers québécois

\begin{tabular}{|l|c|c|}
\hline Usage de... & Classe 1 & Classe 2 \\
\hline Force vis-à-vis d'un récalcitrant & 1,00 & 0,70 \\
\hline Force pour obtenir des aveux & 0,03 & 0,01 \\
\hline Force pour protéger ou se protéger & 1,00 & 0,98 \\
\hline Force pour imposer le respect & 0,25 & 0,06 \\
\hline Arme vis-à-vis d'un récalcitrant & 0,50 & 0,02 \\
\hline Arme pour immobiliser un véhicule & 0,24 & 0,02 \\
\hline Arme pour protéger ou se protéger & 0,99 & 0,97 \\
\hline
\end{tabular}

récalcitrant» ou "pour protéger ou se protéger». Notons que les deux classes sont peu enclines à recourir à la force "pour obtenir des aveux». La même analyse a été répétée à chaque temps de mesure et à chaque fois, le meilleur modèle de classification était celui qui comptait ces deux mêmes classes de répondants. C'est, ici, la proportion de policiers dans chacune de ces classes qui va varier d'un temps de mesure à un autre (tableau 6).

TABLEAU 6

Proportion des policiers québécois par classe en fonction du temps de mesure

\begin{tabular}{|c|c|c|c|}
\hline & T1 & T2 & T4 \\
\hline Classe 1 & $31 \%$ & $35 \%$ & $60 \%$ \\
\hline Classe 2 & $69 \%$ & $65 \%$ & $40 \%$ \\
\hline
\end{tabular}

Alors que les répondants sont tout près de la fin de leur parcours de formation (T1), c'est la deuxième classe qui compte le plus grand nombre de répondants, soit $69 \%$. Nous observons que cette proportion diminue progressivement pour atteindre $40 \%$ lors du dernier temps de mesure. On semble observer ici un phénomène de migration des répondants de la classe 2 à la classe 1 au fur et à mesure que les années passent. 
TA B LEA U 7

Description des classes pour les policiers français

\begin{tabular}{|l|c|c|c|}
\hline Usage de & Classe 1 & Classe 2 & Classe 3 \\
\hline Force vis-à-vis d'un récalcitrant & 1,00 & 0,89 & 0,55 \\
\hline Force pour obtenir des aveux & 0,39 & 0,02 & 0,02 \\
\hline Force pour protéger ou se protéger & 0,99 & 1,00 & 0,22 \\
\hline Force pour imposer le respect & 0,53 & 0,07 & 0,02 \\
\hline Arme vis-à-vis d'un récalcitrant & 0,47 & 0,09 & 0,02 \\
\hline Arme pour immobiliser un véhicule & 0,45 & 0,09 & 0,05 \\
\hline Arme pour protéger ou se protéger & 0,95 & 0,95 & 0,36 \\
\hline
\end{tabular}

La même analyse a été répétée avec les données de l'enquête française. Dans ce cas-ci, cependant, les critères de parcimonie et de qualité d'ajustement pointent vers un modèle à trois classes. Les classes 1 et 2 sont similaires à celles observées chez les Québécois, mais une troisième classe émerge avec les répondants français, soit un groupe pour qui l'usage de la force ou de l'arme semble peu justifié dans presque toutes les situations présentées (tableau 7). Tout comme dans le cas des données québécoises, la même analyse a été répétée pour les trois temps de mesure à peu près équivalents et chaque fois, le modèle comportant ces mêmes trois classes demeurait le meilleur.

TABLEAU 8

Proportion des policiers français par classe en fonction du temps de mesure

\begin{tabular}{|c|c|c|c|}
\hline & T3 & T4 & T5 \\
\hline Classe 1 & $15 \%$ & $36 \%$ & $21 \%$ \\
\hline Classe 2 & $75 \%$ & $60 \%$ & $70 \%$ \\
\hline Classe 3 & $10 \%$ & $4 \%$ & $9 \%$ \\
\hline
\end{tabular}

Tout comme dans le cas des répondants québécois, nous observons ici certaines variations d'une année à l'autre, bien que celles-ci soient beaucoup moins importantes (tableau 8). En effet, à l'exception du fait qu'au deuxième temps de mesure la proportion des policiers faisant partie de la première classe soit plus élevée, les proportions pour chaque classe reviennent peu à peu à des valeurs similaires à celles observées au départ. 


\section{Usage de la force et de l'arme, et l'éthique du métier}

Dix-neuf items du questionnaire utilisé au Québec permettaient l'élaboration d'une échelle de mesure de l'éthique des répondants. Il devenait alors tentant de vérifier quelles allaient être les moyennes de mesure de l'éthique pour les deux classes identifiées en section précédente, selon les différents temps de l'enquête. Ce sont les résultats de cette analyse qui font l'objet du tableau 9. Comme cette analyse ne peut être répliquée à l'étude de Monjardet et Gorgeon, nous pouvons ici présenter les évolutions sur les quatre mesures effectuées.

TABLEAU 9

Moyenne à l'échelle d'éthique par classe pour l'échantillon de policiers québécois

\begin{tabular}{|c|c|c|c|}
\hline & Classe 1 & Classe 2 & $\mathbf{p}$ \\
\hline Temps 1 & 70,7 & 70,8 & 0,905 \\
\hline Temps 2 & 64,9 & 71,1 & $<, 000$ \\
\hline Temps 3 & 63,9 & 69,9 & $<, 000$ \\
\hline Temps 4 & 60,8 & 68,1 & $<, 000$ \\
\hline
\end{tabular}

Au premier temps de mesure, le niveau d'éthique des policiers de la première classe est similaire à celui des policiers de la deuxième classe. La différence entre les deux groupes n'est pas statistiquement significative $(p=0,905)$. Nous observons cependant que la moyenne de la classe 1 diminue graduellement à chaque temps de mesure alors que celle de la classe 2 demeure sensiblement la même. À partir du deuxième temps de mesure, les policiers de la classe 2 affichent significativement une moyenne supérieure à celle des policiers de la classe 1 . Ce résultat suggère que les policiers qui auraient recours à la force ou à leur arme dans une plus grande variété de situations auraient un niveau éthique plus faible que ceux qui réservent l'usage de la force ou de leur arme vis-à-vis d'un récalcitrant ainsi que pour protéger ou se protéger, un usage de la force et de l'arme donc plus restreint.

\section{Discussion}

Bien que quelques similitudes d'opinions ressortent de l'analyse des réponses données par les répondants des deux enquêtes (la question, 
notamment, de la solidarité entre collègues), plusieurs des éléments sur lesquels nous nous sommes arrêtés jusqu'ici montrent deux processus de socialisation professionnelle assez différents dans les deux contextes. De manière générale, dans le cas des répondants français, les opinions et les attitudes se stabilisent peu à peu, un phénomène que Monjardet avait d'ailleurs clairement identifié et qu'il qualifiait de «cristallisation». Force nous est de reconnaître cependant que cette relative stabilisation n'apparaît pas encore dans le cas des recrues policières du Québec. De plus, les manières d'envisager les grands mandats de la police diffèrent passablement, et d'un échantillon à l'autre, et dans le temps au sein de chacun d'entre eux. Notre analyse des raisons du recours à l'arme de service et à la force a montré l'émergence de deux profils distincts chez les répondants québécois, et de trois profils chez leurs homologues français. Or, chez ces derniers, les positions de départ changent assez peu, tandis que les profils des répondants québécois, eux, vont évoluer significativement d'un moment à un autre des premières années de carrière. Dans ce dernier cas, nous avons vu à quel point les répondants qui limitaient le recours à la force à un nombre limité de situations vont peu à peu rejoindre l'autre «camp», soit ceux pour qui l'usage de l'arme et de la force peut se justifier dans un beaucoup plus grand nombre de situations. Et, toujours pour ce qui est des répondants du Québec, nous avons également montré qu'une forme de glissement éthique, vers des niveaux moins élevés d'une phase à une autre, marquait significativement plus ceux et celles que l'on était en mesure de classer comme plus laxistes à l'égard du recours à l'arme et à la force.

En somme, nous nous trouvons à confirmer encore ici davantage l'un des résultats importants de l'enquête Monjardet et Gorgeon:

Le résultat majeur de cette recherche est d'avoir pu vérifier empiriquement - c'était l'une des grandes satisfactions de Dominique - tout à la fois l'existence du pluralisme policier et son évolution dans le temps. [...] l'enquête a indéniablement démontré cette double réalité. (Gorgeon, 2008, cité dans Monjardet, 2008: 242)

Réalité triple, pourrions-nous ajouter, en ce que ces processus vont également diverger d'un contexte organisationnel à un autre, cette dernière observation clouant en quelque sorte un peu plus le cercueil de la thèse de l'unidimensionnalité de la culture professionnelle policière. 


\section{Conclusion}

Il convient, une dernière fois, de revenir sur les limites inhérentes au travail de comparaison que nous avons esquissé au long de cet article, et ce, au-delà des questions plus logistiques et méthodologiques déjà abordées ci-haut. Nous l'avons vu, tout autant au terme de notre courte revue de la littérature pertinente que des résultats exposés, l'idée même de l'existence d'une culture professionnelle policière un tant soit peu universelle ne résiste pas à l'analyse. Or, cet élément constitue d'autant la première et potentiellement la plus importante limite à un travail comparatif. En d'autres termes, ce n'est qu'en articulant la compréhension autour des dispositifs de formation et d'accès à la profession forcément dépendants des traditions, des systèmes scolaires et des administrations que les États font de leur police - que l'on peut mieux arriver à comprendre comment l'apprenant réaménage ses conceptions personnelles du métier de policier au fur et à mesure qu'il en confronte les réalités. Ceci étant dit, il demeure tout de même certains phénomènes plus généraux liés à la socialisation professionnelle, peut-être pas tant dépendants de la profession choisie par l'apprenant que du jeu des réaménagements qu'il aura à faire entre, d'une part, la représentation idéalisée qu'il s'est construite et, d'autre part, la place réelle que les structures d'accueil de sa future profession lui accordent. Ici, le cas de la profession policière est particulièrement éclairant en ce qu'il offre une pléthore de modalités d'accueil - officielles et plus officieuses extrêmement diverses où l'on trouverait à pratiquement ses deux extrêmes les cas de la police française et celui de la police britannique. Cassan (2011) le montre avec force détails: dans le premier cas, l'apprenant est pratiquement considéré comme un poids mort bien encombrant ${ }^{11}$ tandis qu'il s'établit, dans l'autre, une complicité tout à fait remarquable entre la recrue et le mentor qui lui est assigné ${ }^{12}$. Les modalités d'accueil proposées par les organisations policières québé-

11. «L'imprécation virile du commandant («vous êtes des burnes») traduit la position d'infériorité des stagiaires et renvoie à la position de pouvoir détenue par les tuteurs. Le stage est déterminant dans la notation des élèves et pèse sur le classement final, lui-même déterminant sur les affectations futures. Autant d'arguments qui donnent du poids aux menaces proférées au moment de l'accueil. Le stagiaire apprend qu'il n'a pas voix au chapitre et qu'il doit se faire discret» Cassan $(2011: 363)$.

12. «Mais à l'inverse de l'élève français, le probationer [sic] n'est pas laissé à lui-même, son tuteur sert de véritable guide. Se développe ainsi une relation toute particulière entre ces deux personnes, qui ressemble à une forme de compagnonnage» Cassan $(2011: 370)$. 
coises se situent globalement entre ces deux situations pratiquement opposées. Si la recrue policière québécoise ne fait pas l'objet, loin de là, de la sorte d'abaissement que l'on semble retrouver en France, en revanche, la question du mentorat à la britannique n'y est à peu près pas pratiquée officiellement. Plus officieusement toutefois, les longues heures passées à patrouiller en voiture établissent très rapidement un fort climat de complicité entre un nouveau venu et un policier d'expérience. Or, cette complicité porte en elle un potentiel de développement autant d'attitudes «positives» que «négatives». On pourra alors comprendre, par exemple, comme positive toute attitude d'une recrue développant graduellement la position d'un sain réalisme entre les exigences du devoir de servir le citoyen et celles liées au devoir de faire respecter la loi; par contre, beaucoup plus négatif serait le développement d'une attitude de fermeture et de cloisonnement professionnel. C'est dire à quel point, finalement, seuls des travaux de longue haleine sur les mécanismes de socialisation professionnelle des policiers peuvent nous permettre d'établir quels sont ceux qu'il convient de favoriser et, en contrepartie, quels sont ceux pour lesquels des politiques de modulation seraient de mise.

Ceci étant, quels sont donc plus prosaïquement les chantiers qu'il conviendrait d'envisager pour une éventuelle suite des choses? Très clairement, la question des «glissements» éthiques observés chez les répondants québécois appelle son lot de questions. Quels sont ceux de ces nouveaux policiers fraîchement engagés qui «glissent» le plus, et à quel rythme? Y a-t-il des contextes d'intervention policière contextes urbains versus contextes ruraux, pour ne nommer que cette dimension - susceptibles d'accélérer ou de ralentir ce glissement? Les données des deux enquêtes montrent également une division - assez nette chez les Québécois, mais plus nuancée chez les Français - entre ceux et celles qui, d'une part, privilégient l'action comme stimulant de l'intervention policière et ceux et celles qui, d'autre part, vont plus spontanément opter pour l'aide au prochain et le changement social. Or, en partant du principe général que le policier devrait, théoriquement à tout le moins, être en mesure de comprendre qu'il peut et doit faire les deux, la question se pose quant à savoir à quel moment de la carrière une telle jonction s'opère, si jamais elle le fait. Et, finalement, pour reprendre la question que Monjardet a laissée sans réponse, pourrait-on vérifier l'hypothèse d'un éventuel achèvement de la socialisation professionnelle? Telles seraient donc quelques-unes des considérations 
envisageables dans la poursuite de ce type d'enquêtes, au sein de la police bien sûr, mais pourquoi pas également appliquées à d'autres corps professionnels tout aussi symboliquement marqués que le sont les policiers.

\section{Références}

Alain, M., \& Baril, C. (2005a). Attitudes et prédispositions d'un échantillon de recrues policières québécoises à l'égard de leur rôle, de la fonction policière et des modalités de contrôle de la criminalité. Les Cabiers de la sécurité, 58, $3^{\mathrm{e}}$ trimestre, 185-212.

Alain, M., \& Baril, C. (2005b). Crime prevention, crime repression, and policing: Attitudes of police recruits towards their role in crime control. International Journal of Comparative and Applied Criminal Justice, 29 (2), 1-26.

Cassan, D. (2011). Une ethnographie de l'intégration professionnelle du gardien de la paix et du police constable. Déviance et Société, 35 (3), 361-384.

Chan, J. (2003). Fair cop: Learning the art of policing. Toronto: University of Toronto Press.

Conti, N. (2006). Role call: Preprofessional socialization into police culture. Policing ev Society, 16 (3), 221-242.

Conti, N., \& Nolan, J. J. III. (2005). Policing the platonic cave: Ethics and efficacy in police training. Policing $\mathbb{F}$ Society, 15 (2), 166-186.

Coulangeon, P., Pruvost, G., \& Roharik, I. (2012). Les idéologies professionnelles. Une analyse en classes latentes des opinions policières sur le rôle de la police. Revue française de sociologie, 53 (3), 493-527.

Crank, J. (1998). Understanding police culture. Cincinnati: Anderson Publishing.

Dubar, C. (2000). La socialisation: construction des identités sociales et professionnelles. Paris: A. Colin.

Dubar, C. (2010). La socialisation: construction des identités sociales et professionnelles ( $4^{\mathrm{e}}$ éd.). Paris: A. Colin.

Fielding, N. G. (1988). Joining forces: Police training, socialization, and occupational competence. New York: Routledge.

Hogan, T. P., Stephenson, R., \& Parent, N. (2012). Introduction à la psychométrie. Montréal: Chenelière Éducation.

Kappeler, V. E., Sluder, R. D., \& Aplert, G. P. (1998). Forces of deviance, understanding the dark side of policing. Prospect Heights: Waveland Press.

Legault, G. A. (2003). Crise d'identité professionnelle et professionnalisme. Québec: Les Presses de l'Université du Québec.

Malochet, V. (2011). La socialisation professionnelle des policiers municipaux en France. Déviance et Société, 35 (3), 415-438.

Manning, P. K., \& Van Maanen, J. (Eds). (1978). Policing: A view from the street. Santa Monica: Goodyear. 
Marcotte, J., \& Dion, A. (2011). Regards croisés des notions relatives au développement identitaire des jeunes adultes et de la socialisation professionnelle propre au métier de policier. Déviance et Société, 35 (3), 439-449.

McCutcheon, A. L. (1987). Latent class analysis. Quantitative applications in the social sciences (Sage University Paper no. 07-064). Newbury Park: Sage.

McNamara, J. H. (1967). Uncertainties in police work: Recruits' backgrounds and training. Indianapolis: John Wiley and Sons.

McNamara, R. P. (1999). The socialization of the police. In D. J. Kenney \& R. Mcnamara (eds.), Police and Policing: Contemporary Issues (1-12). New York: Praeger.

Monjardet, D. (2008). Notes inédites sur les choses policières. Paris: La Découverte.

Monjardet, D., \& Gorgeon, C. (1992). La socialisation professionnelle: 1167 recruesdescription de la $121^{e}$ promotion des élèves-gardiens de la paix de la police nationale. Paris: Institut des Hautes Études de la Sécurité Intérieure.

Monjardet, D., \& Gorgeon, C. (1993). La socialisation professionnelle des policiers: étude longitudinale de la $121^{e}$ promotion des élèves gardiens de la paix - La formation initiale. Tome I. Paris: Institut des Hautes Études de la Sécurité Intérieure.

Monjardet, D., \& Gorgeon, C. (1996). La socialisation professionnelle des policiers: étude longitudinale de la $121^{e}$ promotion des élèves gardiens de la paix - La titularisation. Tome II. Paris: Institut des Hautes Études de la Sécurité Intérieure.

Monjardet, D., \& Gorgeon, C. (1999). La socialisation professionnelle des policiers: étude longitudinale de la $121^{e}$ promotion des élèves gardiens de la paix - La titularisation. Tome III. Paris: Institut des Hautes Études de la Sécurité Intérieure.

Monjardet, D., \& Gorgeon, C. (2004). La socialisation professionnelle des policiers, dix ans plus tard: la cristallisation. Paris: Acadie-groupe reflex.

Muthen, B., \& Muthen, L. K. (2000). Integrating person-centered and variablecentered analysis: Growth mixture modeling with latent trajectory classes. Alcoholism: Clinical and Experimental Research, 24 (6), 882-891.

Roquet, P. (2012). Comprendre les processus de professionnalisation: une perspective en trois niveaux d'analyse. Phronesis, 1 (2), 82-88.

Sherman, L. (1982). Learning police ethics. Criminal Justice Ethics, 1, 10-19.

Van Maanen, J. (1973). Observations on the making of policemen. Human Organisation, 32 (4), 407-228.

Van Maanen, J. (1974). Working the street: A developmental view of police behaviour. In $\mathrm{H}$. Jacob (ed.), The potential for reform of criminal justice. Beverly Hills: Sage.

Van Maanen, J. (1977). Organizational careers: Some new perspectives. New York: John Wiley.

ABSTRACT - Professional socialization is known as the study of how newly trained professionals integrate their chosen profession and its most dominant cultural traits. Generally, professional socialization studies rely on the testimonies of those more or less experienced in the profession who had lived through such a process. Researching 
and documenting professional socialization processes while they occur, are nonetheless rare. And it is even less frequent that such endeavors have been replicated in two different countries for one same profession. In this article present research conducted in France and Quebec on the professionalization of police recruits. Both studies use equivalent questionnaires and procedures and were conducted within a ten years time span. Analysing and comparing the two studies at equivalent moments will give the readers glimpses at more or less generic processes, while also enlighten the differences attributable to the specific professional contexts within which socialization occurs.

KEY WORDS - professional socialization, professional culture, police culture, international comparison, police ethics.

RESUMEN - La cuestión del reajuste de las representaciones que un aprendiz hace de su oficio, forma parte ahora de un corpus de conocimientos conocido bajo el nombre de socialización profesional. De forma general, es a posteriori que dichos reajustes, sus amplitudes y sus formas son estudiados, analizando los discursos de los representantes de una o de varias profesiones. Es, en efecto, mucho más raro de documentar estos reajustes en tiempo real, o sea, siguiendo el recorrido de socialización en el transcurso en el que éstos se desarrollan. Igualmente, es aún más raro que un dispositivo de investigación longitudinal sobre cohorte sea objeto de una replicación en otro contexto. Dicho ejercicio ha sido realizado con diez años de intervalo en Francia, lugar originario de la encuesta, y en Québec a continuación. Si bien el tratar de realizar una comparación entre los contextos francés y quebequense del oficio de policía, no constituye nuestro primer objetivo, el hecho de disponer de dos recopilaciones de datos de encuestas de socialización profesional diferentes nos posibilitara el surgimiento de procesos genéricos en común, de aquello que parece ser más especí fico a uno o al otro de los dos contextos.

PALABRAS CLAVE - Socialización profesional, cultura profesional, cultura policial, comparación internacional, ética policial. 